\title{
Outdoor Image Segmentation Method Utilizing Color Cluster Elimination and Texture Sewn Response
}

\author{
Hossein Abbasi ${ }^{1}$, Salwani Mohd Daud ${ }^{1}$, Nilam Nur Amir Sjarif ${ }^{1}$ and Morteza \\ Abbasi $^{2}$

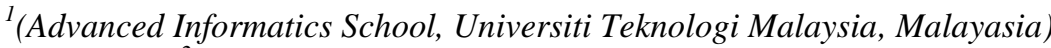 \\ ${ }^{2}$ (Isfahan University of Technology, Isfahan, Iran)
}

\begin{abstract}
Colour clustering is one of the colour reduction methods applicable for segmentation. Nevertheless, it has not been an appropriate method cause of colour and texture variety of outdoor image. Hence, customizing segmentation is necessary for outdoor image which can improve the quality of computer vision system to a large extent by recognizing the objects. This study focuses on customizing the colour clustering methods for segmentation and object recognition in the outdoor images by utilizing a multi-phase procedure through a multi-resolution platform, called Colour Cluster Elimination (CCE), where, the primary colour clusters are detected and then gradually eliminated, allowing the smaller clusters to emerge in much clearer versions of the image. Afterward, each colour region is examined to identify the adjacent homochromatic objects with different textures. A new texture feature: Texture Sewn Response (TSR) has been introduced to indicate the texture strength in different direction/resolutions. The strength has been shown by combining the energies of the detail images applying the Gabor filters bank. Finally, the proposed method has been applied for evaluating on outdoor images dataset namely BSDS500 and the results have been compared to PRI, NPR, and GCE statistical metrics of the latest segmentation methods which demonstrated that the proposed method has a satisfactory performance for the segmentation of the outdoor scenes.
\end{abstract}

Keywords: Image Segmentation, Outdoor Image, Color Cluster, CCE, Texture Sewn Response.

\section{Introduction}

Outdoor scenes processing and analysis have gained a special importance in computer vision systems in recent years. They have the most applications in traffic control [1], security organization [2], automated analysis of the images of cities [3], mobile robots [4], and wearable computers designs [5], assistance of nearly blind or blind people for travel [6]. The increase in the number of applications of the computer vision enhances the importance of the development of this system. The first phase of the vision system is segmentation. Segmentation is one of the most important bottom-up processing of images that continuously describes the existing objects in the image. The purpose of segmentation is simplifying and changing the image presentation in a way that is more meaningful and easier for analysis [7] by creating regions that are different in features while the pixels of each region share the similar features.

The quality of segmentation has a great impact on the process of objects recognition. Colour as one of the main low-level feature of images contributes to the quality of segmentation. One of the most important tools for image segmentation is using the colour characteristics of the pixels. The purpose of colour clustering of the images, which is sometimes called colour reduction is merging the similar colours and creating a new image with a limited number of colours. In colour clustering methods we are looking for the dense places in a colour space. Each place stands for primary colours or similar ones. Each of the dense places or pre patterns represent an object homochromatic objects, in addition, low-impact colours merge with the important colours of the image and reduce the processing load of the colour segmentation algorithm. Several methods have been proposed to reduce the colours. A set of algorithms use statistical clustering methods to find an optimal colour box [8] such as GNC [9], FOSART [10] and FUZZY ART [11]. A simple, efficient and fast way for clustering is using kmeans algorithm [12]. The algorithm and its fuzzy version, fuzzy c-means or FCM have many applications in image processing and machine learning. Some algorithms have been proposed in recent studies [13, 14]. Neural networks are also used for clustering colour features. For instance, Kohonen's self-organizing map [15] is popular for reducing the colours and it has been frequently adopted [16-18].

Colours clustering can be useful in merging sub patterns and representing homo-chromatic objects [19]. However usual clustering methods based on partitioning the colour space or statistical patterns (e.g. kmeans and neural networks) have failed to establish appropriate pre-patterns for segmentation and object recognition in outdoor images $[20,21]$. In other words, while we expect the colour clusters developed from the reduction process represent important objects and patterns in outdoor images, some extracted pre-patterns have shown that the related colours contain more pixels. 
On the other hand, the problems of the clustering algorithms by the aim of appropriate segmentation pre patterns creating in the outdoor images consist of over segmentation and under segmentation. Over segmentation occurs when an object is partitioned into several smaller segments, while under segmentation occurs when just one colour is attributed to some single objects. In reducing the colours of an outdoor image, there are two problems. Due to the large number of pixels in the bigger clusters, the clustering algorithms focus more on such clusters and this leads to over segmentation. Conversely, it makes tiny objects stay unnoticed and under segmentation occurs. As a result, the clustering algorithms should be customized to be suitable for the segmentation process. By using an optimized colour clustering, each region includes an object or a pattern which all its sub-regions have the similar colours. However, there is a possibility that some objects are placed next to each other and corresponded to a region by the colour clustering algorithm [22]. Therefore, this region needs a texture segmentation to differentiate homochromatic adjacent regions which have dissimilar texture. In 2013 Rasti et al. proposed a new colour reduction method via colour clustering in which 9 clusters where obtained by Gradual Clustering Elimination. However, this method had some drawbacks considering the extra created clusters and disregarding textures of images [23].

Aforementioned discussion shows that, colour clustering method optimization is necessary for outdoor image segmentation and for this reason, a new customized method based on the colour clustering of images along with the use of texture features namely Texture Sewn Response (TSR) has been presented in this paper, which has assured the good quality for outdoor image segmentation. TSR methods have a superior capability for indicating the texture strength in different direction/resolutions for outdoor image.

\section{Typical Methods Of Segmentation And Segmentation Quality Evaluation}

Successful recognition of objects depends upon the segmentation quality of the images and various methods have been proposed and analysis by the researchers for the segmentation of outdoor images. Here, CCE and TSR procedures for image segmentation are discussed in details in the followings as these two method could contribute significantly in the field of image processing. In addition, various methods are suggested for segmentation quality evaluation and this study evaluates the quality of the proposed algorithm on three indexes: PRI, NPR, GCE.

\subsection{The CCE procedure}

Common colour clustering algorithms have the limitations of performing segmentation of outdoor scene like wrong identification of outdoor scene and wrong detection of objects. However, these methods can be improved by customizing the segmentation methods of outdoor scene colour clustering. In the top-levels of the multi resolution pyramid, the texture details and tiny objects are faded. As a result, colours are the most important feature of the objects for segmentation. Moreover, the colour shades of an object are merged to a large extent. These two features provide a perfect platform to reduce outdoor colour images. Figure 1 shows Multi resolution pyramid for an outdoor image, where, in each level of pyramid, using clustering, and multi resolution images can recognize important objects and then remove them from the lower levels of the pyramid. This way, the segmentation continues to be focused on the remaining images. This method compared to a complete and one phase image clustering at different levels of pyramid shows a better performance. The reason is that it prevents the effects of big clusters on colour clustering at the bottom levels of pyramids.

The stages of this method that henceforth is called CCE for creating a six colour image using two levels of pyramids are as follows:

1. Using a smoothing filter, two blurred versions of the image that have been respectively called, B1 and B2 emerged. Figure 2 represents the two blurred images along with the original one.

This filtering process helps to eliminate those things (the infuriating points, tiny objects and texture details which are mainly observed in the outer images) that raise problems for segmenting. Moreover, by combining similar colours, only the important colour categories will remain.

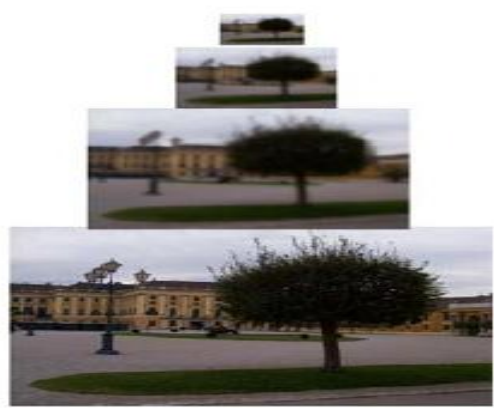

Figure 1. Multi Resolution Pyramid for an Outdoor Image 
2. Using the k-means algorithm, the image B2 (the most blurred version of the image) is divided into three colour clusters. Figure 3(a) shows an outdoor image and figure 3(b) illustrates the 3 coloured version of the image. These three clusters correspond to very important colours of the images, which have high pixel density. An important point is that there are the numbers of pixels, which are not really similar to the three clusters, or they belong to other objects and their colours are different from the colour of the three clusters but they are forced to be in the clusters. The next phase will be clustering these points separately.

3. Now we will calculate the Euclidean distance of the points of the image B1 to the three centroids obtained from the previous step. Those points that their Euclidean distance of three centroids is greater than the th 1 threshold, don't fall into the colour clusters and need to be re-clustered again. Later, we will explain how the threshold is determined. The points in figure 4 are called orphan pixels. The white parts of the image are non-orphan pixels. They have found their appropriate centroids in the first phase of clustering.

4. Similar to the method adopted in phase 2, we will divide the orphan points into three new clusters to have a total of 6 colour clusters in the image.

5. Then, we will compare the image pixels with the obtained centroids of the clusters. Each pixel that shares more similarity with the cluster centroids will be attributed to that cluster.

The obtained clusters in the image which were divided into 6 classes (Figure 3) are more suitable for segmentation than those classes which came out of the initial division of the image. For a better comparison, in the figure, we have used a different colour palette and flashy colors for pseudo-coloring.

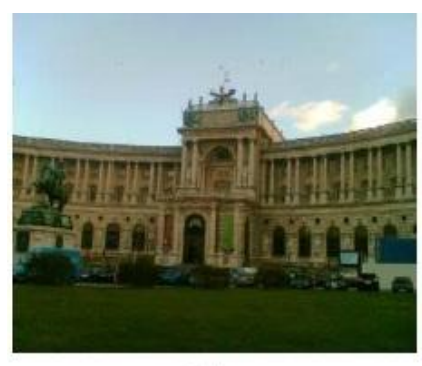

(a)

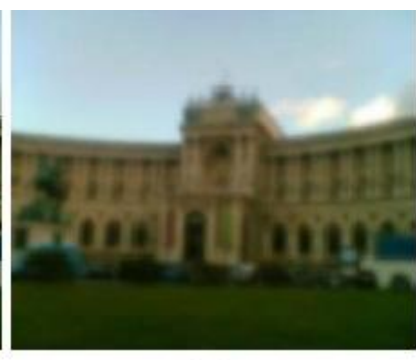

(b)

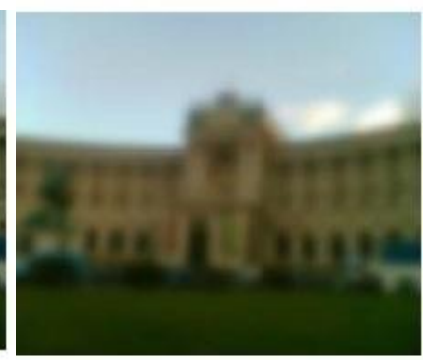

(c)

Figure 3. The Blurred Versions of an Outdoor Image (a) The Original Image,

(b) The Image B1 (c) The Image B2

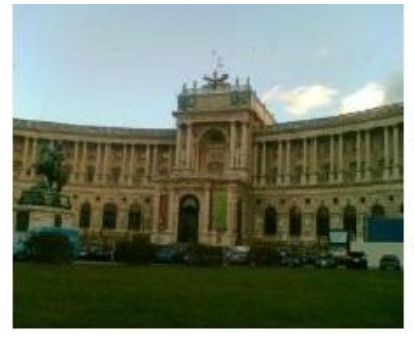

(a)

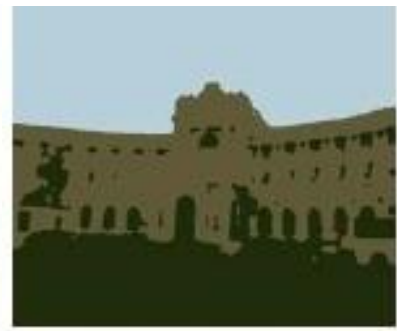

(b)

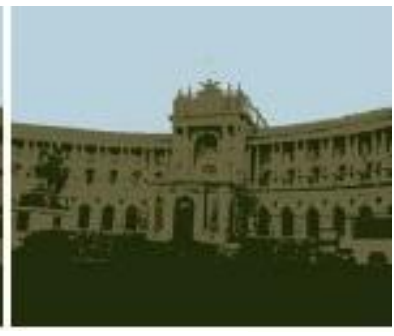

(c)

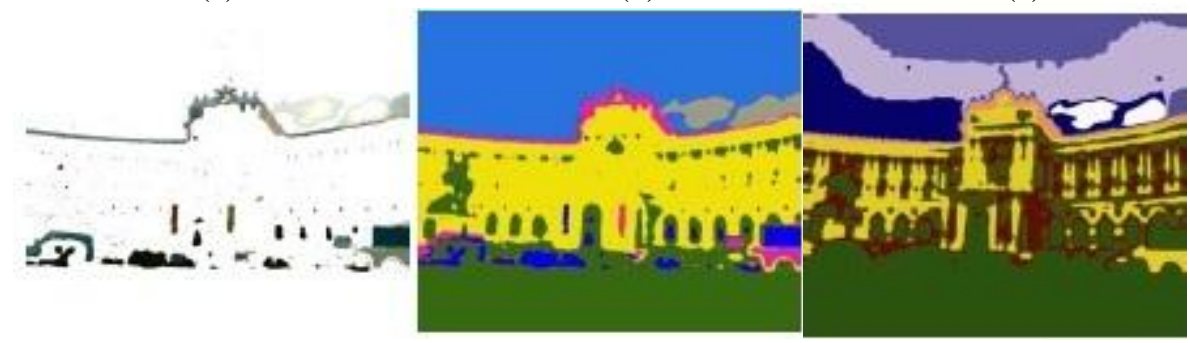

(d)

(e)

(f)

Figure 3. The Effects of Standard Clustering on an Outdoor Image (a) The Original Image (b) The ThreeColored Version of B2, (C) the Original Colored Image, (d) The Pixels that are not placed in the Three Colour Clusters (Orphan Pixels), (e) The Six-Colored Image created by the Multi Resolution Pyramid, (f) the SixColored Image Created by Standard Clustering.

\subsection{The TSR procedure}

TSR is an abbreviation of texture sewn response in which The term sewn refers to the calculation method of TSR feature, as the different responses of the Gabor filters are combined weightily (sewn), and create the feature. The numerical computation of the features is as follows: 
First we will calculate the frequency signature vector of all pixels, $\left(\mathrm{V}=\left[\mathrm{V}_{\mathrm{i}} \mid 1 \leq \mathrm{i} \ll 16\right]\right)$ Element $\mathrm{Vi}$, in the set (the vector) $\mathrm{V}$ of each pixel stands for the energy of that pixel in resolution and direction of i. It is obtained after applying the Gabor filters. The element V 1 and element V16 are respectively assumed in the direction of $0^{\circ}$ of the first octave in the direction of $135^{\circ}$ of the fourth octave.

The mean of vectors $\mathrm{V}$ for all pixels are calculated and is called $\mathrm{G}$. The $\mathrm{i}$ th member of the vector $(\mathrm{Gi})$ is the result of applying the Gabor filter i (related to a specific resolution and direction) on the image pixels.

Based on the members of vector $\mathrm{G}$, vector $\mathrm{V}$ will be given a threshold value and converted to zero and one to obtain a set of vectors $\mathrm{T}$. The vector $\mathrm{T}$ is obtained by thresholding the vector $\mathrm{V}$ according to the (1).

$$
T_{i}= \begin{cases}0 & v_{i} \leq G_{i} \\ 1 & v_{i} \geq G_{i}\end{cases}
$$

In this equation, $\mathrm{Vi}$ is the $\mathrm{i}$ th element of the vector $\mathrm{V}$ and $\mathrm{Ti}$ is the $\mathrm{i}$ th of element of the vector $\mathrm{T}$ corresponding to it; in simple words, each element of the vector $\mathrm{V}$ is related to a pixel (indicates the energy of the pixel in the resolution and direction of i) is compared with the average energy of all the pixels in that direction and resolution and if it is less than the average amount it will be zero but if the amount is greater than the average it, it will be 1 . At the end of the phase, each image pixel has a gray-tone energy vector $\mathrm{T}$ in which zero represents low energy and 1 stands for high energy in the corresponding resolution and direction. For example, if T1 is a pixel which is equal to zero, the energy of that pixel in the first octave, and in the direction 0 is lower than the average energy of all pixels in the same resolution and direction. Figure 4 shows the thresholding of a vector V(right) using the average vector G (Inside the thresholding block) and creating the gray-tone energy vector $\mathrm{T}$ (lift).

For measuring TSR, the same procedure used for LBP will be adopted. Equation (2) shows how to calculate TSR feature for a pixel with a gray-tone energy vector. Since the energy level of each pixel in different directions and resolution is shown using the gray energy-tone vector T, by multiplying the elements of the vector $\mathrm{T}$ by the integer powers of 2 and summing them together, we will obtain a set of weighted summation of the energies, for different resolutions and regions patterns which are unique. At the end of the process, each pixel will have a TSR number. Obviously, clustering the set of numbers is easier than the multi-dimensional clustering of the signature frequency vectors.

$$
S G R=\sum_{i=1}^{16} T_{i} 2^{i-1}
$$

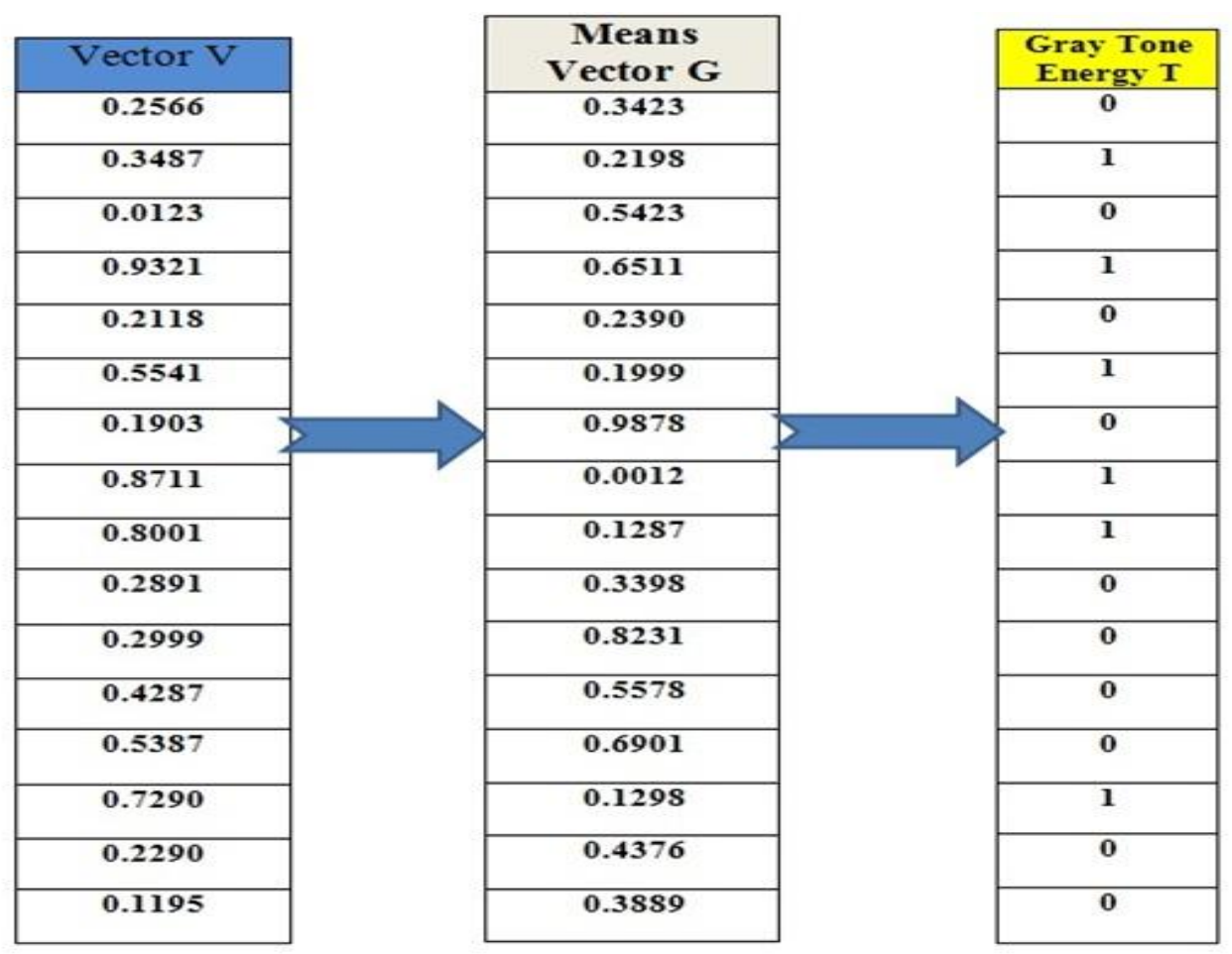

Figure4: Thresholding the Gabor energies and Creating Gray-Tone Energy Vector. 
Outdoor Image Segmentation Method Utilizing Color Cluster Elimination and Texture Sewn..

\subsection{PRI Index}

The index is used for evaluating the quality of the segmentation in the statistical algorithms [24]. It examines pair or identical elements in the two clusters, imagine we have the reference image $(G)$ and the segmented image (S). The sum of the pairs of pixels which have the same labels in $\mathrm{G}$ and in $\mathrm{S}$ (in each of the two images are part of a cluster) are called TP. In other words, if the pair pixels of $i$ and $j$ of the image have the same labels in both $\mathrm{G}$ and $\mathrm{S}$, they will fall in TP category. The sum of the pairs of pixels which have the same labels neither in $\mathrm{G}$ nor in $\mathrm{S}$ (in each of the two segmentations are not part of a cluster) are called TN. The sum of the pairs of pixels which don't have the same labels in G (are not in a cluster), whereas they share the same labels in S (are in a cluster) are called FP. The sum of the pairs of pixels which have the same label in G (are in a cluster), whereas they don't share the same labels in S (are not in a cluster) are called FN.

$\mathrm{RI}$ index is the proportion of $\mathrm{S}$ and $\mathrm{G}$ pairs that have been labeled properly to the total number of pairs which is shown in (2).

$$
R I=\frac{T P+T N}{T P+T N+F P+F N}
$$

The value of PRI is between zero and one if image $\mathrm{S}$ is different from the reference image (image $\mathrm{S}$ has one segment and the images of the reference set include the segments of the size of a pixel), the amount of PRI would be zero and in the case of the entire similarity between S and reference segments, the value of PRI would be equal to 1 .

\subsection{The NPR index}

It is one of the common measure of the quality assessment of clustering algorithms [25]. It has been used in many studies, however due to the low dynamic range, the small difference between the clustering algorithms are not shown. In this study, the image segmentation were randomly generated and compared with the Berkeley database, the amount of the PR would be equal to 0/63. It means that a variation range would fall between 0.63 to an amount less than one. In [25] the standardized version of the PR is introduced under the name of the NPR. Equation (3) shows how the NPR index is calculated. Calculating NPR in this way makes its average zero and its range wider. Clearly such a wide range makes NPR more sensitive than PRI, meaning that small changes in the algorithm performance will cause drastic changes in NPR

$$
N P R=\frac{\text { Index }- \text { Expected Index }}{\text { Maximum Index }- \text { Expected Index }}
$$

\subsection{GCE Metric}

It is one of the most important metrics to evaluate the quality of a region-oriented segmentation, general compatibility error or GCE [26]. This metric specifies the extent to which one segmentation is the modified version of the other. Comparing the two segmentations, the metric shows the error is trivial and both segmentation is compatible and have classified the image to different detailed levels. Suppose $\mathrm{R}(\mathrm{S}, \mathrm{Pi})$ is a region of the segmentation $\mathrm{S}$ that includes pixel Pi. If both segmentation $\mathrm{S} 1$ and $\mathrm{S}-2$ of an image is presented, the local refinement error is defined as (4) where $\mathrm{R}$ indicates set difference and A stands for the number of set members.

$$
\operatorname{LRE}\left(S_{1}, S_{2}\right)=\frac{\left|R\left(S_{1}, P_{i}\right) / R\left(S_{2}, P_{i}\right)\right|}{\left|R\left(S_{1}, P_{i}\right)\right|}
$$

It is clear that if the segment of $\mathrm{S}$ which contains the pixel $\mathrm{P}$ in $\mathrm{S} 1$, is a subset of pi in $\mathrm{S} 2$, the error is equal to zero. In other words, this section can be considered as a modification of segmentations. On the other hand, the different segmentations will cause large errors (Equation 5 shows how to calculate the global consistency error).

$$
\operatorname{GCE}\left(S_{1}, S_{2}\right)=\frac{1}{n} \min \left\{\sum_{i} E\left(S_{1}, S_{2}, P_{i}\right), \sum_{i} E\left(S_{2}, S_{1}, P_{i}\right)\right\}
$$

This error in some cases is defined [0-1] the less the error is, the more similarity is shared between S1 and S2. To assess the quality of the proposed algorithm, we will evaluate it on the Berkeley database images and calculate the average of CGE metric for the segmentations of the reference of each image, and then we calculated the average of this measure for all the datasets.

\section{Texture Segmentation by Clustering the Features of TSR}

For TSR features clustering, we can use the idea of self-organizing map of the neural networks [27]. In the networks, the training process begins with few neurons and if more neurons are needed, they can be 
generated from the neurons at the boundary and grow the network. The proposed steps of the procedure are as follows:

1. We will find the features of TSR which is mostly repeated in the image (the TSR histogram mode). The pixels with the TSR comprises of the primary texture.

2. We will find the second most frequent TSR (the second peak of the TSR histogram). The pixels with the TSR value can create a new texture. In order to ensure that the texture is new, the Euclidean distance of frequency signature vector of the image pixels, which have this amount of TSR, need to be compared with the frequency signature vector of the old texture. If the Euclid distance is greater than the given threshold, we are dealing with a new texture and need to register it; otherwise the new pixels will attach the old texture.

3. In each phase, we will find the highest unprocessed peak of TSR histogram (see the methods in phase 2). Then we will examine whether we are dealing with a new texture or not. Considering the texture, we will check if the pixels with the same amount of TSR are similar to one of the clusters which have already been registered and need to attach them. The process continues to the point where the highest peak in the histogram of TSR is very short (unclassified pixels belong to less important textures of the image).

4. We will compare the frequency signature of UN clustered pixels with the frequency signature of the registered images by calculating the Euclidian distance. Then we decide to attribute each pixel to the appropriate texture. As it is shown there is no need to determine the number of clusters (textures) before clustering and the main clusters will emerge during the process. Figure 5 shows the block diagram of the proposed method block diagram.

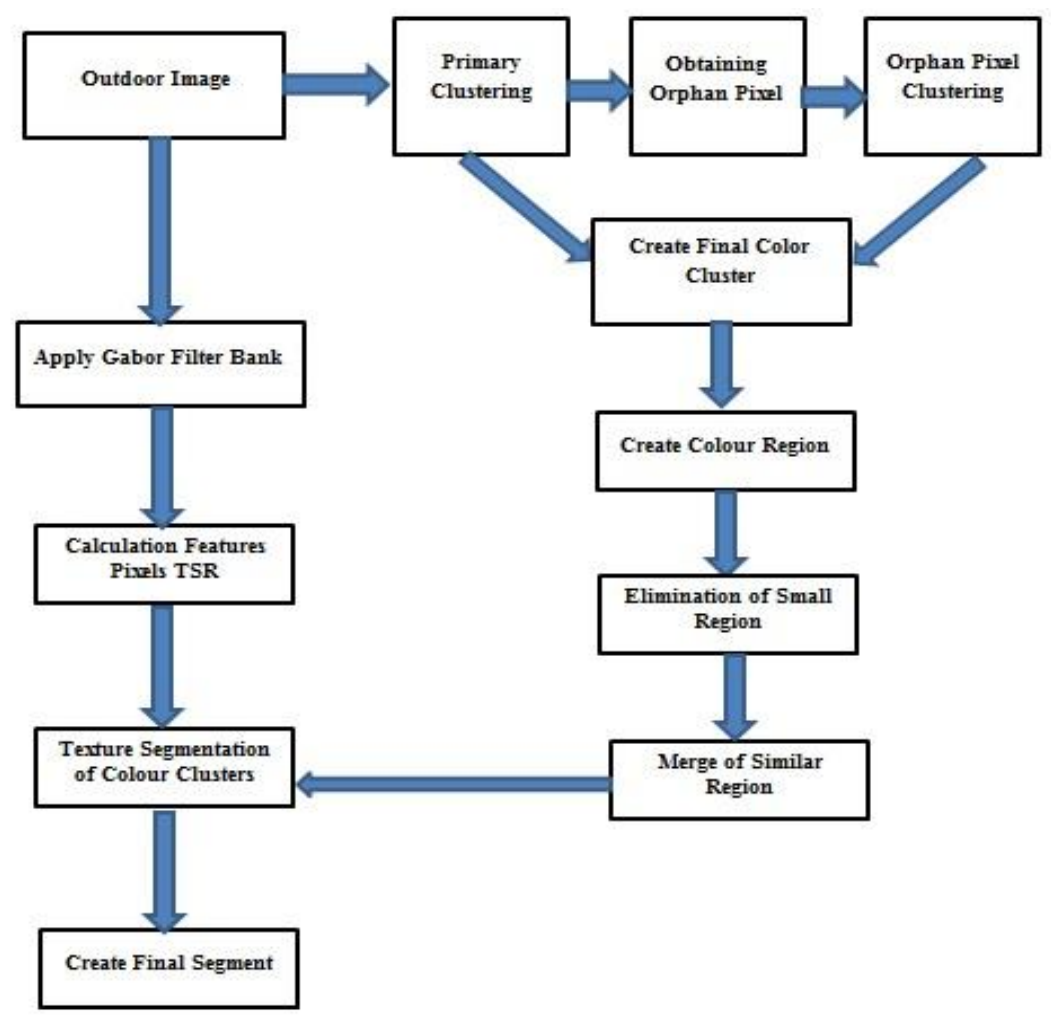

Figure 5. Block diagram of the working principle of the proposed method

\section{Empirical evaluation:}

Proposed method has been evaluated on several clustered images on the Berkeley dataset which is shown in figure 6. As it is shown in this figure, in the preliminary phase of image clustering, the colours of adjacent areas are merged with the similar colours. However, by applying the texture analysis, it is possible to separate the distinct regions in a colour region. Segmentation quality will be analyzed by using three wellknown quantitative metrics by the following procedure:

1. The PRI index is between [0-1]. If two segments are quite different, the RI is " 0 ". This happens when the whole image is considered as a segment and each pixel of the image as another segment. If two segments are quite similar, the PRI is " 1 ". Thus much closer RI (between the segmented image and the reference image) to 1 leads to the more quality and precision of the algorithm. 
2. GCE metric specifies the extent to which segmentation is the modified version of the other segmentation. Comparing the two segmentations, the metric shows the error is trivial and both segmentation are compatible and have classified the image to different detailed levels.
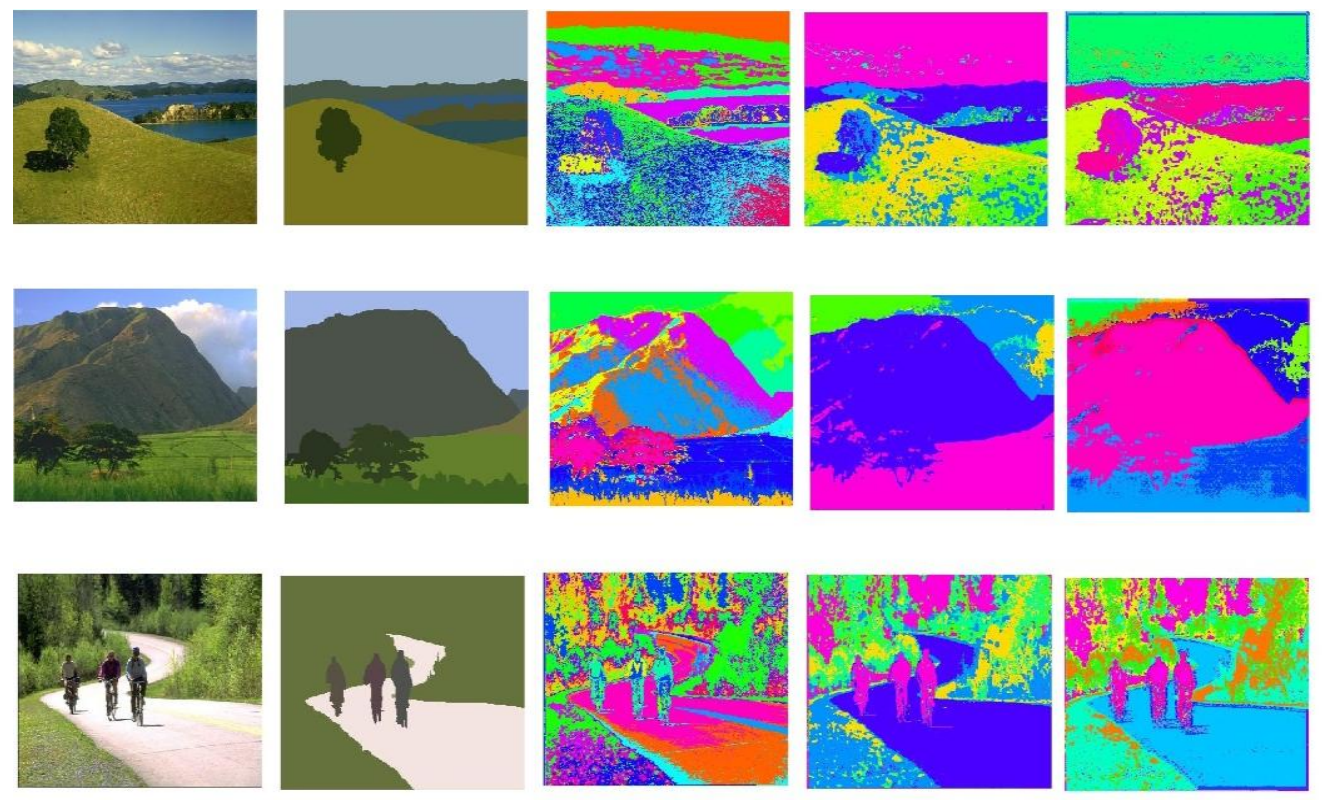

(a)

(b)
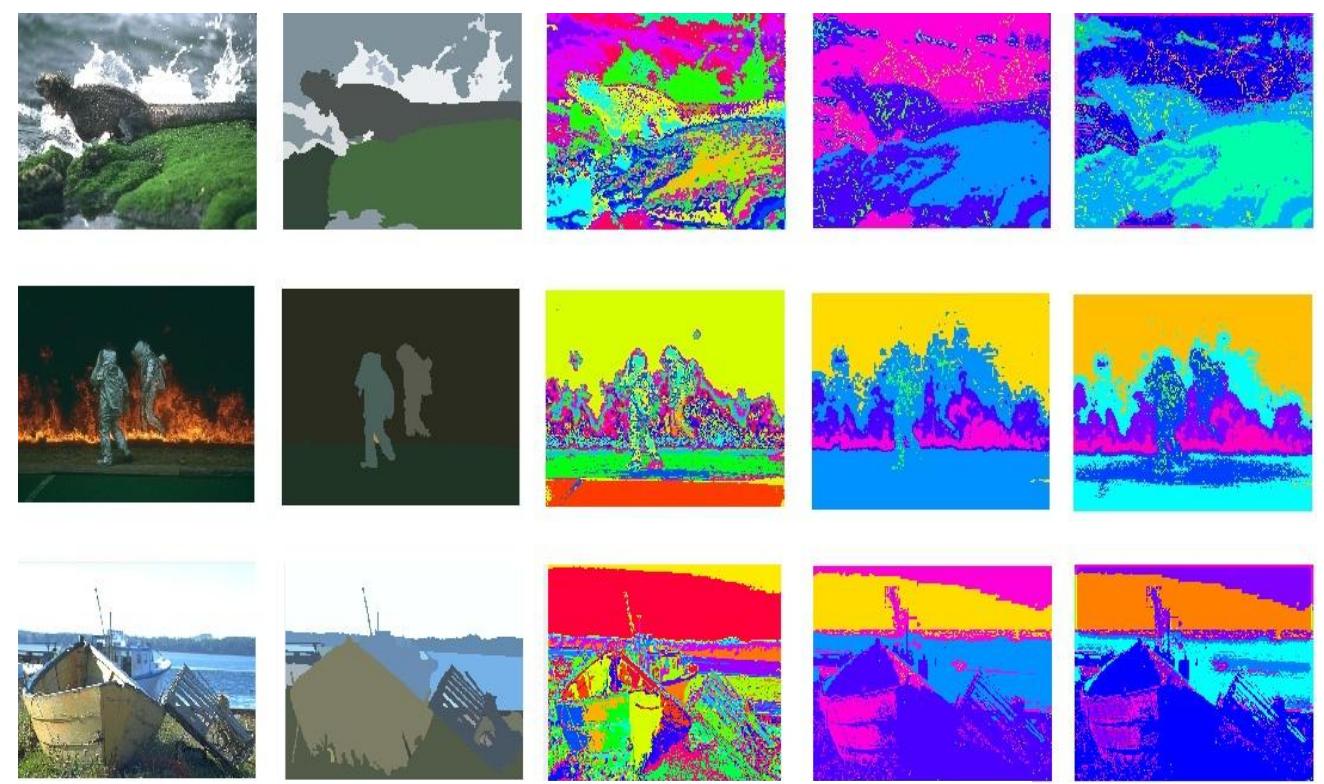

(c)
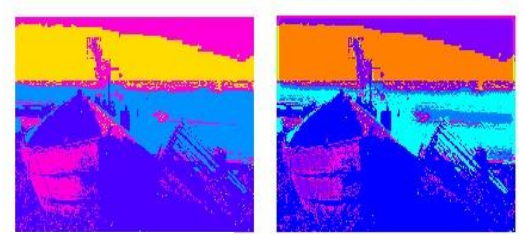

(d)

Figure 6: Some examples of color images of BSDS300 dataset which are clustered based on. (a) The original image, (b) The ground truth image, (c) The color clustered image by k-means, (d) The color clustered by Hue method, (e) The color clustered image by TSR method.

\section{Experimental Result, Analysis And Discussion}

Applying this method, the segmentation algorithm was evaluated on the datasets images of BSDS300. The measures were obtained for the output images. The results in (obtained by Using PRI, NPR and GCE metrics) showed that the proposed algorithm was as high-quality as known region feature low-level segmentation algorithms and the comparison of the results for various methods are shown in Table 1.

The method which proposed in this study is effective for the images with large clusters (such as landscape or scenery), while the Berkeley dataset includes different images such as animals, human faces and 
urban landscapes that are not appropriate for the proposed method. For example, by selecting 50 images of BSDS dataset 300 which have large clusters and are kinds of landscape, PRI metric will increase to $83 \%$.

Table1: Comparison of the standard clustering and the gradual elimination based clustering performance on the BSDS300 dataset.

\begin{tabular}{|c|c|c|c|c|}
\hline Clustering algorithm & Standard clustering & Trimmed mean & Histogram analysis & Proposed method (Hue+TSR) \\
\hline PRI & 0.72 & 0.77 & 0.79 & 81 \\
\hline NPR & 0.32 & 0.49 & 0.52 & 55 \\
\hline GCE & 0.36 & 0.29 & 0.25 & 18 \\
\hline
\end{tabular}

In order to propose the appropriate patterns of segmentation, we face the challenges of oversegmentation and under- segmentation in colour clustering of the outdoor images. Such challenges can be eliminated the colour cluster elimination based algorithm which resolves the over-segmentation issue by limiting the number of clusters in the first phase and prevents the under-segmentation issue by focusing on the smaller clusters in the second phase. Features of the gradual elimination based algorithm are as follows:

1. Effective Management of the details: Small objects and texture details do not disturb the clustering process. The reason is that they will be removed in the initial phase through the soothing process then will emerge as time passes. Using the multi resolution pyramid, the results are improved noticeably. Without the pyramid, the segmentation error will increase for $6 \%$ [28]

2. Appropriate for the Conditions of Outdoor Images: Compared to the standard clustering, the gradual elimination based clustering method is more resistant to inappropriate conditions of the outdoor images for instance similar colour shades and luminosity effects. In other words, in the standard clustering, the number of pixels affects the centroids. For this reason, there are some shades correspond to a colour with a large number of pixels in an image, the shades are considered as different objects. In the proposed method, in each stage those objects that have quite a large number of pixels or several colour shades will be considered as a single object and eliminated. In the next phase, the focus will be on the remaining pixels. Therefore, not only the shades of a colour are treated as a single object but also those segments which are eliminated in the standard method will be shown in the proposed method.

3. Low Quality in Inappropriate Colour Conditions: Colour clustering does not show appropriate quality in the images with similar colours (compared to other methods which only use the low level futures of images) and this algorithm shows a better quality for the images with the big clusters (e.g. landscape).

4. Resistant to Noise: The multi resolution context used for the algorithm prevents the negative influence of noise to be imposed on the segmentation of colour clustering. Figure 7 illustrates the effect of adding white noise with the ratio of signal to noise five and ten decibels on the performance of different algorithms on the Berkeley dataset images.
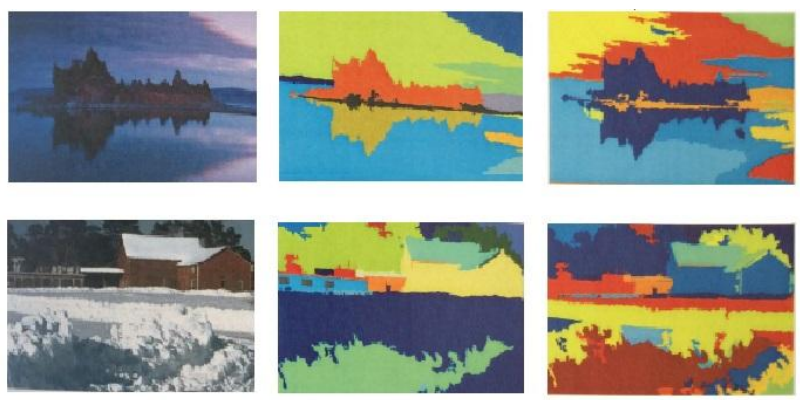

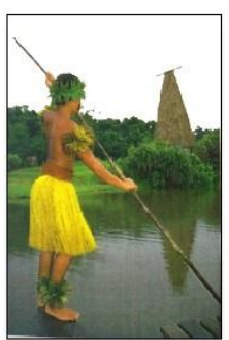

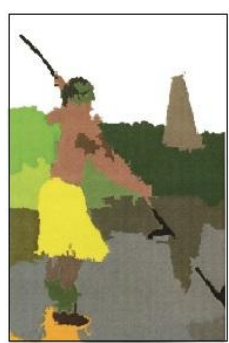

(b)

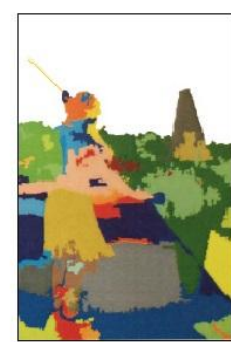

Figure 7: The effect of adding white noise on the performance of the segmentation algorithms. The right column from top to down: the original image, the noisy image $\mathrm{SNR}=5 \mathrm{db}$, the noisy image $\mathrm{SNR}=10 \mathrm{db}$; the middle column: the result of the proposed algorithm; the left column: the result of Mean Shift algorithm 


\section{Conclusion}

Inspired by the human visual system, a multi-phase method called CCE in the context of multiresolution images was proposed. In each phase of the method, the important colour clusters related to the large objects of the images were recognized and gradually eliminated to allow the smaller clusters emerge in clearer versions. The proposed clustering method has shown a better performance than the standard clustering for object recognition. The reason is that it prevents the pixels density of large clusters from affecting the clustering structure. The major clusters in each phase were detected using the statistical methods based on the trimmed mean and analyzing the color histograms. In addition, the morphological functions were used to eliminate the existing sub regions which remain after clustering within the large regions. The weighted Graph theory is employed to estimate the primary colours of the images, and merge the similar adjacent regions. The emerged segments applying the CCE algorithm are merely based on the colour features of images. However, in colour clustering, two diverse homochromatic objects, which have different textures, may be located next to each other and form a cluster. This explains why each of the emerged colour regions needs to be re-examined and in the case they do not share identical texture in various parts, they need to be partitioned into some different sub regions. The emerged detailed images after applying the Gabor filters bank are textually analyzed. For each pixel $n$-number vector called frequency signature is obtained. The general guideline for identifying different texture using frequency signature vectors is analyzing the distribution of the vectors and clustering them. However, the clustering in the $\mathrm{n}$-dimensional domain is difficult.

To tackle this problem, we introduced a new feature of texture, or TSR which combines the thresholding and gray -tone energies of the Gabor detail image and introduces a weighted summation for each pixel that indicates the combined energies in different directions and resolution. For texture analysis of a colour region, the amount of TSR of all pixels belonging to the region will be calculated. Using this method, in addition to reducing the computational load of multi-dimensional clusters, appropriate precision is also guaranteed. For evaluating the algorithm, it has been tested on dataset BSDS300 and the results have been compared with well -known segmentation algorithm based on quantitative criteria such as GCE, NPR and PRI.

\section{References}

[1] J. T. Lee, M. S. Ryoo, M. Riley, and J. K. Aggarwal, "Real-Time Illegal Parking Detection in Outdoor Environments Using 1-D Transformation," IEEE Transactions on Circuits and Systems for Video Technology, vol. 19, pp. 1014-1024, Jul 2009.

[2] P. M. Ciarelli, E. O. T. Salles, and E. Oliveira, "Human automatic detection and tracking for outdoor video," Integrated ComputerAided Engineering, vol. 18, pp. 379-390, 2011.

[3] G. Monteiro, J. Marcos, M. Ribeiro, and J. Batista, "Robust segmentation for outdoor traffic surveillance," in 15th IEEE International Conference on Image Processing, 2008, pp. 2652-2655.

[4] C.-K. Chang, C. Siagian, and L. Itti, "Mobile Robot Vision Navigation Based on Road Segmentation and Boundary Extraction Algorithms," Journal of Vision, vol. 12, pp. 200-200, 2012.

[5] M. R. Everingham, B. T. Thomas, and T. Troscianko, "Wearable mobility aid for low vision using scene classification in a Markov random field model framework," International Journal of Human-Computer Interaction, vol. 15, pp. 231-244, 2003.

[6] C. L. Chen, Y. F. Liao, and C. L. Tai, "Image-to-MIDI mapping based on dynamic fuzzy color segmentation for visually impaired people," Pattern Recognition Letters, vol. 32, pp. 549-560, Mar 12011.

[7] S. Ahmed, Z. Khan, and A. Jain, "Image Segmentation by using Mean-Shift with Dynamic Region Merging-A Survey," International Journal of Advanced Research in Computer Science and Electronics Engineering (IJARCSEE), vol. 1, pp. pp: 268$273,2012$.

[8] N. Vlajic and H. C. Card, "Vector quantization of images using modified adaptive resonance algorithm for hierarchical clustering," IEEE Transactions on Neural Networks, vol. 12, pp. 1147-1162, Sep 2001.

[9] B. Fritzke, "A growing neural gas network learns topologies," Advances in neural information processing systems, vol. 7, pp. 625632,1995

[10] A. Baraldi and P. Blonda, "A survey of fuzzy clustering algorithms for pattern recognition - Part I," IEEE Transactions on Systems Man and Cybernetics Part B-Cybernetics, vol. 29, pp. 778-785, Dec 1999.

[11] G. A. Carpenter, S. Grossberg, N. Markuzon, J. H. Reynolds, and D. B. Rosen, "Fuzzy ARTMAP: A neural network architecture for incremental supervised learning of analog multidimensional maps," IEEE Transaction on Neural Network, vol. 3, pp. 698-713, 1992.

[12] R. O. Duda, P. E. Hart, and D. G. Stork, Pattern classification: John Wiley \& Sons, 2012.

[13] Y. C. Hu and M. G. Lee, "K-means-based color palette design scheme with the use of stable flags," Journal of Electronic Imaging, vol. 16, pp. 033003-033003-11, Jul-Sep 2007.

[14] P. Ng and C.-M. Pun, "Skin color segmentation by Texture Feature Extraction and K-mean Clustering," in Third International Conference on Computational Intelligence, Communication Systems and Networks, 2011, pp. 213-218.

[15] M. Oja, S. Kaski, and T. Kohonen, "Bibliography of self-organizing map (SOM) papers: 1998-2001 addendum," Neural computing surveys, vol. 3, pp. 1-156, 2003.

[16] R. M. D. M. Venkatesan, "IMAGE SEGMENTATION BY FUZZY C MEANS CLUSTERING: SURVEY."

[17] A. Atsalakis and N. Papamarkos, "Color reduction and estimation of the number of dominant colors by using a self-growing and self-organized neural gas," Engineering Applications of Artificial Intelligence, vol. 19, pp. 769-786, Oct 2006.

[18] K. Zagoris, N. Papamarkos, and I. Koustoudis, "Color reduction using the combination of the Kohonen self-organized feature map and the Gustafson-Kessel fuzzy algorithm," in Machine Learning and Data Mining in Pattern Recognition, ed: Springer, 2007, pp. 703-715.

[19] G. Dong and M. Xie, "Color clustering and learning for image segmentation based on neural networks," IEEE Transaction on Neural Network, vol. 16, pp. 925-36, Jul 2005. 
[20] R. Huang, N. Sang, D. Luo, and Q. Tang, "Image segmentation via coherent clustering in L* a* b* color space," Pattern Recognition Letters, vol. 32, pp. 891-902, 2011.

[21] R. Figueiredo, L. Schnitman, and J. A. F. de Souza, "Using neural network and k-means cluster for image segmentation in outdoor scenes," Revista Ciências Exatas, vol. 14, 2009.

[22] M. Blas, M. Agrawal, A. Sundaresan, and K. Konolige, "Fast color/texture segmentation for outdoor robots," in IEEE/RSJ International Conference on Intelligent Robots and Systems, 2008, pp. 4078-4085.

[23] J. Rasti, A.M., and A. Vafaei, "Gradual Cluster Elimination For Color Clustering In Outdoor Scenes," International Journal of Innovative Computing, Information and Control, vol. 9, pp. 2441-2464, 2013.

[24] J. Rasti, S. A. Monadjemi, and A. Vafaei, "A graph-based vision system for automatic object detection in outdoor scenes," Annals of DAAAM \& Proceedings, pp. 167-169, 2011

[25] R. Unnikrishnan, C. Pantofaru, and M. Hebert, "Toward objective evaluation of image segmentation algorithms," IEEE transactions on pattern analysis and machine intelligence, vol. 29, pp. 929-944, 2007.

[26] D. Martin, C. Fowlkes, D. Tal, and J. Malik, "A database of human segmented natural images and its application to evaluating segmentation algorithms and measuring ecological statistics," in Eighth IEEE International Conference on Computer Vision, 2001, pp. 416-423.

[27] D. E. Ilea and P. F. Whelan, "CTex - an adaptive unsupervised segmentation algorithm based on color-texture coherence," Image Processing, IEEE Transactions on, vol. 17, pp. 1926-1939, 2008.

[28] A. M. Javad Rasti, Abbas Vafaei, "GRADUAL CLUSTER ELIMINATION FOR COLOR CLUSTERING IN OUTDOOR SCENES," International Journal of Innovative Computing, Information and Control, vol. 9, pp. 2441-2464, 2013. 